\title{
TarMed-Informationen für die Ärzte / Informations sur le TarMed pour des médecins
}

Die Seminarteilnehmer erhalten Einblick in den neuen Tarif «TarMed» und Gelegenheit, in einem Workshop Fallbeispiele zu lösen. Für FMH-Mitglieder ist die Teilnahme am Seminarunterricht kostenlos, für Nicht-FMH-Mitglieder wird ein Beitrag von Fr. 300.- erhoben.

Les participants au séminaire seront informés sur le nouveau tarif médical TarMed. Ils auront aussi la possibilité, en ateliers, de résoudre des problèmes particuliers. La participation au séminaire est gratuite pour les membres de la FMH et coûte Fr. 300.- pour les non-membres.

Seminar/séminaire:

Nr. 28 deutsch Samstag, 24. Juni 2000 Lugano 08.30-12.00 Uhr Hotel de la Paix

$\mathrm{N}^{\circ} 29$ français samedi, 24 juin $2000 \quad$ Lugano $13.30-17.00 \mathrm{~h} \quad$ Hôtel de la Paix

\author{
Anmeldung/Inscription \\ Ich melde mich hiermit für das folgende Seminar an: \\ Je m'inscris au séminaire suivant: \\ Seminar $\mathrm{Nr}$. $/ \mathrm{N}^{\circ}$ du séminaire

\begin{tabular}{ll} 
Name/Nom & Vorname/Prénom \\
\hline Strasse/Rue & PLZ/Ort / NPA/Lieu \\
\hline Tel. privat / Tél. privé & Tel. Praxis/Spital / Tél. cabinet/hôpital \\
\hline Datum/Date & Unterschrift/Signature
\end{tabular} \\ Telefonische Auskünfte erhalten Sie unter Tel. 0419250077 bei Frau S. Lichtsteiner \\ Renseignements complémentaires: Mme S. Lichtsteiner, tél. 0419250077 \\ Die Anmeldung ist möglich per Telefon, Post, E-mail oder Fax an: \\ Vous pouvez vous inscrire par téléphone, par bulletin d'inscription, par fax ou e-mail: \\ FMH Tarifdienst, Frau S. Lichtsteiner, Burghöhe 1, Postfach 246, 6208 Oberkirch, Fax 04192105 86, E-mail fmhtarif@hin.ch \\ Aus organisatorischen Gründen bitten wir Sie, sich frühzeitig anzumelden. Die Teilnehmerzahl ist begrenzt. \\ Pour des raisons d'organisation, nous vous prions de vous inscrire le plus tôt possible. Le nombre de participants est limité.
}

